\title{
Diatoms of Bagmati River, Kathmandu, Nepal
}

\author{
Shiva Kumar Rai ${ }^{*}$ and Sudip Khadka \\ Phycology Research Lab \\ Department of Botany, Post Graduate Campus, T.U., Nepal \\ *E-mail: sk.khaling@gmail.com
}

\begin{abstract}
This paper is an outcome of M.Sc. dissertation work carried out by second author during 2014 in Bagmati River, Kathmandu, Nepal. Diatom samples were collected by scraping stones with the toothbrush, squeezing macrophytes and sucking by dropper from three different sites (Mulkharka, Gokarna, Guheshwori-Pashupatinath) along the river during summer, rainy and winter seasons and studied thoroughly in the Phycology Researh Laboratory, Department of Botany, Post Graduate Campus, Biratnagar. A total 48 diatom taxa belonging to 25 genera and 12 families were enumerated. Among them, 20 diatoms were reported first time from Nepal viz., Fragilaria vaucheriae, Achnanthes inflata, Planothidium lanceolatum, Eunotia botuliformis, Gyrosigma scalproides, Stauroneis smithii, Sellaphora capitata, Neidium ampliatum, Navicula escambia, Pinnularia cf divergens, P. grunowii, Gomphonema acidoclinatum, G. gandhii, G. pseudoaugur, G. rhombicum, Cymbella turgidula, Encyonema hustedtii, E. ventricosum, Epithemia adnata, and Surirella angusta. Further extensive studies are essential to understand more about new and interesting diatoms of this river.
\end{abstract}

Key words: Bacillariophyceae, Gomphonema, Pashupatinath, Planothidium Sellaphora, Sundarijal

\section{Introduction}

Diatoms (Bacillariophyceae) are a large group of microscopic algae inhabited in almost all aquatic or wet environments throughout the globe. They have unique shells made up of silica and are most beautiful among the microscopic objects due to wonderful sculpturing of their cell walls (Prescott, 1969). They are mostly unicellular but some exist in colony and a few also exhibit filamentous habit. The shapes of diatom varies greatly ranging from oval, rounded, elongated, conical, rod shaped, disc shaped to triangular. The estimated number of diatoms exist in the world is more than 200,000 taxa (Mann \& Droop, 1996).

Diatoms are very common and diverse group of algae and are considered as major producer of organic matter. They contribute to $25 \%$ of the atmospheric oxygen, and approximately $43 \%$ of the primary production of the oceans (Treguer et al., 1995). They are good bioindicators, as diatom assemblages quickly respond to environmental changes, due to their relatively short life spans and rapid immigration rates (Prygiel et al., 1999). Diatoms can also be applied in general aquatic bio-assessment, which uses species richness, composition and abundance to assess human impacts on aquatic environments, and global changes in biodiversity (Stoermer \& Smol, 1999). Their wall with silica deposition is very useful to nanotechnology (Bradbury, 2004). Diatom cells repeatedly and reliably manufacture valves of various shapes and sizes, potentially allowing diatoms to manufacture micro or nano scale structures which may be of use in a range of devices, 
including optical systems: semiconductor, nanolithography and even using diatom valves as vehicles for drug delivery. Using an appropriate artificial selection procedure, diatoms that produce valves of particular shapes and sizes could be evolved in the laboratory, and then used in chemostat cultures to mass produce nanoscale components (Drum \& Gordon, 2003). Besides these, diatoms could also be used as a component of solar cells, by substituting photosensitive titanium dioxide for the silicon dioxide normally used in the creation of cell walls (Johnson, 2009). Diatom biofuel producing solar panels have also been proposed (Ramachandra et al., 2009). Diatoms can also be used in forensic researches especially in drawn cases.

Habitat destruction and eutrophication threaten many diatom species with extinction. The most threatened taxa are believed to be those that occur only in restricted habitats and are usually found in low numbers. Diatoms are reported to be very sensitive to global temperature, particularly to the equator-pole temperature gradient. Warmer oceans in the past have been shown to have had substantially lower diatom diversity. Future warm oceans enhanced by global warming could thus result in a significant loss of diatom diversity (IPCC, 2007). Realizing various beneficial utilities of diatoms, numerous taxonomic studies on diatoms have been carried out in the globe.

In Nepal, diatoms studies were very scanty. Diatom of Kathmandu valley was studied by few workers at different intervals of time. Hirano (1963) reported 33 species of diatoms from ponds at Tahachal, Baudhanath, Dillibazar, Ranipokhari at Kathmandu; and Patandhoka at Lalitpur. Four species of diatoms have been reported from Nagdaha and Taudaha, Kathmandu (Hickel, 1973). Shrestha and Manandhar (1983) have also reported 4 species of diatoms from Kathmandu. The most important work in Kathmandu valley was carried out by Simkhada and Jüttner (2006) who reported 213 species of diatoms from Taudaha, Chobhar; Dhungapokhari, Kirtipur; Thankot; Balaju water garden, Kathmandu; Malpokhari near Bagdol; pond at Jawalakhel Zoo; Botanical garden, Godawari; Ranipokhari, Ratnapark; Narayan pokhari, south gate of Palace; Siddhapokhari and Kamalpokhari, Bhaktapur. The fossil diatoms of Kathmandu basin also have been studied by Hayashi et al. (2007, 2012), Hayashi (2011), Hayashi and Tanimura (2015a, b) reporting 36 species including 5 new taxa from the core at Rabibhawan. Diatoms of Bagmati River was least studied. Shrestha and Manandhar (1983) and Simkhada and Jüttner (2006) have reported few species of diatoms from Sundarijal, Bagmati river. Very few are known about the diatoms of Bagmati River. Thus, an endeavor is made to explore the diatom of Bagamati River at different localities in different seasons.

\section{Materials and Methods Study area}

The Bagmati River is originated from Baghdwar $\left(27^{\circ} 46^{\prime} 16^{\prime \prime} \mathrm{N}, 85^{\circ} 25^{\prime} 38^{\prime \prime} \mathrm{E}\right)$ which lies above the southern edge of the Shivapuri Hills about $15 \mathrm{~km}$ northeast of Kathmandu. Here, the Bagmati is wide and swift with a high load of suspended solids, giving it a grey appearance. The river flows southwest about $10 \mathrm{~km}$ through terraced rice fields in the Kathmandu Valley. Resistant rock strata interrupt the flow in places, including at Pashupatinath temple. Beyond the temple, the river flows south until joined by the larger west-flowing Monahara river, then turns west itself. After entering Kathmandu's urban area 
more tributaries enter: relatively unpolluted Dhobi Khola and sewage-laden Tukucha Khola. Then the river bends south and the Vishnumati enters from the right at Teku Dovan. The Vishnumati also rises from the Shivapuri Hills, about $6 \mathrm{~km}$ west of the Bagmati's source. It flows south passing Nagarjun Hill and Forest Reserve, Swayambhu Stupa and Durbar Square in Kathmandu. As it passes throught the centre of Kathmandu, this tributary becomes heavily polluted and choked with trash. Flowing generally south although with many curves, the Bagmatai reaches the edge of the Kathmandu Valley and enters Chobar Gorge near the Dakshinkali temple complex. The gorge cuts through the Mahabharat Range or Lesser Himalaya. The Bagmati also crosses the lower Sivalik Hills before reaching the Terai, and then crosses into India at Dheng.

Kathmandu is situated in or near the subtropical wet forest biome. The average annual temperature is $18.3^{\circ} \mathrm{C}$. In the urban center, the temperature fluctuates between $32^{\circ} \mathrm{C}$ in summer (June-July) to $-2^{\circ} \mathrm{C}$ in winter (December-January). In the winter, day temperatures reach $19.7^{\circ} \mathrm{C}$ on average falling to $2^{\circ} \mathrm{C}$ overnight. In spring, temperatures climb reaching $27.3^{\circ} \mathrm{C}$ generally in the afternoon with overnight lows of $11^{\circ} \mathrm{C}$. During summer average high temperatures is $28.3^{\circ} \mathrm{C}$ and average low temperatures is $19.3^{\circ} \mathrm{C}$. Total average annual precipitation is $1343 \mathrm{~mm}$.

\section{Sample collection and identification}

Diatom samples were collected in summer, rainy and winter seasons, 2014, from 3 different sites of Bagmati River: Site 1 was Mulkharka $\left(27^{\circ} 46^{\prime} 45.02^{\prime \prime} \mathrm{N}, 8^{\circ} 25^{\prime} 27.31^{\prime \prime} \mathrm{E}\right.$, alt. $1674 \mathrm{~m}$ $\mathrm{msl}$ ), about $1 \mathrm{~km}$ north from Sundarijal Water Reservoir, lies in Sundarijal Gokarneshwor Municipality (Figs. A, D-F); site 2 was Gokarna $\left(27^{\circ} 44^{\prime} 6.64^{\prime \prime} \mathrm{N}, 85^{\circ} 24^{\prime} 17.83^{\prime \prime}\right.$ E, alt. $1325 \mathrm{~m}$ msl) where the river just enter into the valley (Fig. B); and site 3 was GuheshworiPashupatinath area $\left(27^{\circ} 42^{\prime} 37.43^{\prime \prime} \mathrm{N}, 8^{\circ} 21^{\prime} 15.99^{\prime \prime}\right.$ E, alt. $\left.1306 \mathrm{~m} \mathrm{msl}\right)$, Kathmandu (Fig. C).

In each trip, 30 samples were collected for each site. Samples were collected carefully and any potential contamination was avoided. For each site, a different set of materials (bottles, brush, dropper etc.) were used in order to avoid contamination and mixing up of diatom species. The epilithic diatoms were scraped out using toothbrush, the epipelic forms were collected using dropper, and the epiphytic forms were squeezed out from the roots and leaves of aquatic macrophytes. The materials were then preserved in FAA solution as soon as collected to prevent the decomposition of diatoms. Samples were labeled properly including collection number, date of collection, site number, season and habitats. Water temperature and $\mathrm{pH}$ were measured with the help of Hanna Digital meter. Geographical positions (latitude, longitude and elevation) of each collection spot were noted with the help of GPS Garmin e-Trex. Necessary photographs of the diatom habitats were captured with the help of Canon Digital Camera. Field notes including habitat nature, water pollution level, surrounding vegetation were also maintained properly in the field note book. Then, the samples were brought to the Phycological Research Laboratory, Department of Botany, Post Graduate Campus, Biratnagar for further detail investigation.

The preliminary screening of samples were made to confirmed the occurrence of diatoms by observing temporary glycerine mount slides under light microscope and unnecessary blank samples were discarded. Selected samples were checked for preservatives, if necessary, preservative was added, then stored in refrigerator under $20-25^{\circ} \mathrm{C}$. 

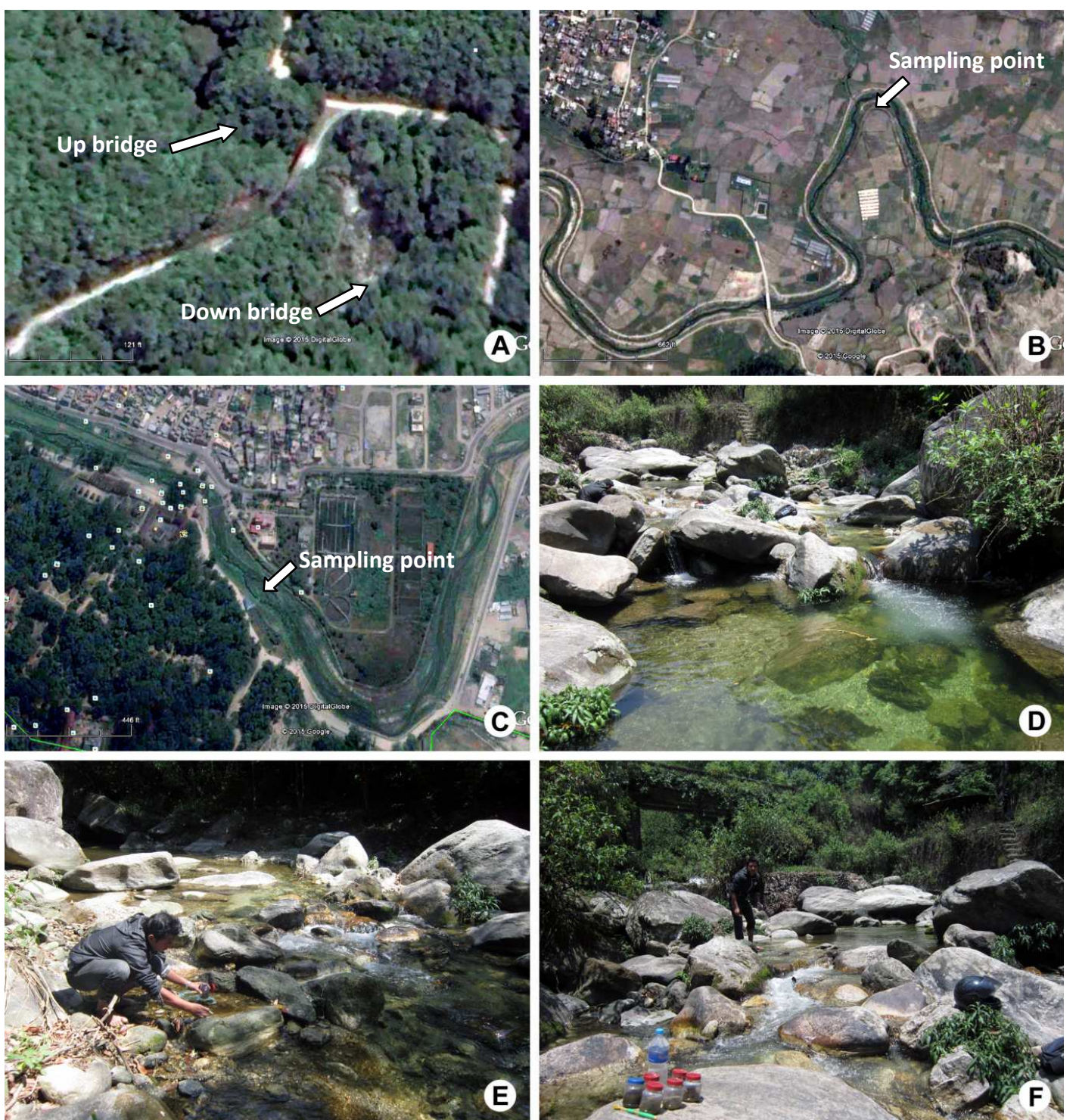

Figures: A. Up and down bridge sampling points at Site 1, Bagmati river, Mulkharka B. Site 2 of Bagmati river at Gokarna C. Site 3 of Bagmati river at Guheshwori-Pashupatinath D-F. Collecting diatoms in Bagmati river at Mulkharka.

Diatom frustules were cleaned following the modified method of PCER, ANSP (1988). The slides were examined using compound light microscope under $20 \mathrm{X}$ and $40 \mathrm{X}$ magnifications. Photomicrographs were taken with the help of Olympus CH20i trinocular microscope attached with digital camera under objectives 40X and 100X magnifications. Diatoms were identified consulting various literatures and monographs such as Tiffany and Britton (1952), Lange-Bertalot (1980, 1993, 1996, 2001), Krammer and Lange-Bertalot (1986, 1988, 1991), Prasad and Srivastava (1992), Lange-Bertalot amd Metzeltin (1996), Krammer (1997, 2002), Mann et al. (2004), Metzeltin et al. (2005, 2009), Rai (2006), Metzeltin and Lange-Bertalot (2007), Lange-Bertalot et al. (2011), Karthick et al. (2013), 
etc. All the collected materials and slides have been deposited in the repository of Phycological Research Lab, Department of Botany, Post Graduate Campus, Biratnagar.

\section{Results and Discussion}

A total 48 diatom taxa belonging to 25 genera and 12 families were reported from three different sites of Bagmati river in summer, rainy and winter seasons. The taxonomic treatment for each taxon is given below.

\section{Aulacoseira granulata (Ehr.) Simonsen (Fig. 1)}

Reference: Karthick et al. 2013, Pl. 3.

Characters: Frustules joined end to end by long spines to form afilament; smaller spines also present around the valve margin; valves in girdle view with many coarse areole; areole are concentrated to the margin; valve diameter $12-29 \mu \mathrm{m}$, valve mantle depth 3.5-6 $\mu \mathrm{m}$, striae 8-12 in $10 \mu \mathrm{m}$.

Habitat: Site 1, Sundarijal, epilithic.

Collection no. \& date: Sundarijal, summer 10; 2014-06-10.

Distribution in Nepal: A ditch at Pitchara canal, Morang (Rai \& Rai, 2005); Phewa, Rupa and Begnas lakes, Pokhara, Kaski (Nakanishi, 1986); Punyamati river, Kavrepalanchok (Aryal \& Lacoul, 1996).

\section{Cyclotella meneghiniana Kutz. (Figs. 2-5)}

References: Prasad and Srivastava 1992, P. 160, Pl. 24, Figs. 1-2; Karthick et al. 2013, Pl. 5; Agata 2009, P. 135, Pl. 1, Fig. 14-15.

Characters: Frustules drum-shaped; radially symmetrical, marginal striae coarse, wedge shaped; central zone smooth; valve diameter 11-35 $\mu \mathrm{m}$; striae 5-9 in $10 \mu \mathrm{m}$.

Habitat: Site 1, Sundarijal, epilithic; Site 3, Pashupati, epilithic.

Collection no. \& date: Sundarijal, summer 10; 2014-06-10; Pashupati, rainy 3; 2014-8-25.

Distribution in Nepal: Pond at Dillibazar-1,300m, Kathmandu (Hirano, 1963).

3. Diatoma hiemale var. mesodon (Her.) Grun. (Figs. 6-9)

References: Tiffany and Britton 1952, P. 230, Pl. 61, Fig. 685; Hirano 1971, Pl. 4, Figs.1516; Rai 2006, P. 71, Pl. 4, Figs.12-13, 16-17; Agata 2009, P. 138, Pl. 3, Fig. 15-19.

Characters: Valves joined in zigzag chains; each valve broadly elliptical, rectangular; tabular in girdle view; transverse striations; costae prominent, finely punctuate striations between the costae; 11-39 $\mu \mathrm{m}$ long, 6.5-16 $\mu \mathrm{m}$ broad.

Habitat: Site 1, Sundarijal, epilithic, epipelic, epiphytic; Site 2, Gokarna, epilithic.

Collection no. \& date: Sundarijal, summer $1,2,5,6,9$; 2014-06-10, rainy 1, 2, 5, 6; 20148-22 and winter 3, 4, 6, 8; 2015-2-15; Gokarna, rainy 3; 2014-08-23.

Distribution in Nepal: A stream west from Dudh Koshi river-5,250 m, Solukhumbu, Eastern Nepal (Suxena \& Venkateswarlu, 1968); a stream at Wolang Chung Gola (Hirano, 1984).

4. Meridion circulare var. constrictum (Ralfs) Hustedt. (Fig. 10)

Reference: Tiffany and Britton 1952, P. 228, Pl. 61, fig. 691.

Characters: Valves wedge shaped, constricted at broader end, larger pole broadly capitates; transverse septa rudimentary, appearing often as costae on valves and girdles; 
fine transverse striations between the costae; $10-80 \mu \mathrm{m}$ long, $4.5-8 \mu \mathrm{m}$ broad, striae $12-15$ in $10 \mu \mathrm{m}$.

Habitat: Site 1, Sundarijal; Stream with running water, epipelic.

Collection no. \& date: Sundarijal, summer 14; 2014-06-10.

Distribution in Nepal: Pond at Arughat Bazar, Gorkha 770m. (Hirano, 1963).

5. Fragilaria vaucheriae Kutz. Petersen (Fig. 11)

References: Lange-Bertalot 1980, Pl. 1, Figs. 26-34; Rumrich et al. 2000, Pl. 3, Figs. 7-13.

Characters: Valves lineo-lanceolate to linear; apex generally slightly capitates; narrow and linear longitudinal area with a pseudo-raphe; central area from one edge to the other of the valve; parallel striations becom slightly radiant to the ends; $10-48 \mu \mathrm{m}$ long, $4.5-5.5 \mu \mathrm{m}$ broad, striae 9-13 in $10 \mu \mathrm{m}$.

Habitat: Site 3, Pashupati, epilithic.

Collection no. \& date: Pashupati, rainy 1; 2014-8-25.

Distribution in Nepal: Not reported earlier from Nepal.

6. Ulnaria ulna (Nitzsch) Compere (Figs. 12-16)

References: Karthick et al. 2013, Pl. 18.

Characters: Valves linear, with parallel margins; central sternum narrow straight; single labiates process located near the apex of one end of the valve; striae parallel; 72-105 $\mu \mathrm{m}$ long, 6-8 $\mu \mathrm{m}$ broad, striae 7-10 in $10 \mu \mathrm{m}$.

Habitat: Site 1, Sundarijal, epilithic, epipelic, epiphytic; Site 2, Gokarna, epilithic, epipelic; Site 3, Pashupati, epilithic.

Collection no. \& date: Sundarijal, summer 2, 7, 8; 2014-6-10; rainy 1, 5; 2014-8-22; winter 6, 9; 2015-2-15; Gokarna, rainy 2, 6; 2014-8-23; Pashupati, rainy 1; 2014-8-25.

Distribution in Nepal: A stream at Kungbachen, Panchthar- 4150m. (Hirano, 1984).

7. Achnanthes crenulata Grun. (Figs. 17-24)

References: Rai 2006, P. 81, Pl. 7, Figs. 4-5.

Characters: Valves linear, elliptical to narrow elliptical, sides crenulate, broadly rounded end; hypovalve with thin median raphe with distinct central nodules; axial area narrow widen towards the centre; central area medium; girdle view curved; striae coarse, punctuate, radial towards the end; epivalve with linear narrow, marginal pseudoraphe; striae coarsely punctuate, parallel at middle, slightly curved towards the end; 33-43 $\mu \mathrm{m}$ long, 12 $14 \mu \mathrm{m}$ boad, striae 8-10 in $10 \mu \mathrm{m}$.

Habitat: Site 1, Sundarijal, epilithic, epipelic, epiphytic; Site 2, Gokarna, epilithic, epipelic; Site 3, Pashupati, epilithic.

Collection no. \& date: Sundarijal, summer 1, 6, 8; 2014-6-10; rainy 2, 4, 5; 2014-8-22; winter 3, 5, 6, 9; 2015-2-15; Gokarna, rainy 1, 2, 6; 2014-8-23; Pashupati, rainy 2, 3; 2014$8-25$.

Distribution in Nepal: A stream at Kungbachen, Panchthar- 4,150 m, Eastern Nepal (Hirano, 1984).

8. Achnanthes inflata (Kutz.) Grun. (Figs. 25-26)

References: Rai 2006, P. 82, Pl. 7, Fig. 8-10; Alfinito and Lange-Bertalot 2013. P. 139, Figs. 62-64; Karthick et al. 2013, Pl. 23.

Characters: Valves linear with two strong concave undulations forming gibbous centre and two long broadly rounded ends; hypovalve with thin, straight median raphe with 
distinct central nodules; axial area narrow gradually widening towards centre; central area broad, stauroid reaching the sides; striae coarse, punctuate, radial; 46-47 $\mu \mathrm{m}$ long, 7.5-12.5 $\mu \mathrm{m}$ broad, striae 13 in $10 \mu \mathrm{m}$.

Habitat: Site 1, Sundarijal; epiphytic; Site 3, Pashupati, epilithic.

Collection no. \& date: Sundarijal, rainy, 5; 2014-8-22; Pashupati, rainy 2, 3; 2014-8-25.

Distribution in Nepal: Not reported earlier from Nepal.

9. Planothidium lanceolatum (Breb.) Round and Bukhtiyarova (Figs. 27-31)

References: Agata 2009, P. 142, Pl. 6, Figs. 8-17.

Characters: Valves lanceolate with sub-rostrate ends; central area asymmetrical with a hood on internal valve; raphe straight with expanded external end; striae slightly radiate throughout the valve; $14.5-30 \mu \mathrm{m}$ long, $6.5-9.5 \mu \mathrm{m}$ broad, striae $10-12$ in $10 \mu \mathrm{m}$.

Habitat: Site 1, Sundarijal, epilithic, epipelic, epiphytic.

Collection no. \& date: Sundarijal, summer 2, 6, 8; 2014-6-10; rainy 4, 5; 2014-8-22; winter 3, 5, 7, 9; 2015-2-15.

Distribution in Nepal: Not reported earlier from Nepal.

10. Cocconeis placentula Ehr. (Fig. 32)

References: Tiffany and Britton 1952, P. 241, Pl. 64, Fig. 736; Prasad and Srivastava 1992, P. 199, Pl. 27, Fig 6. Agata 2009, P. 143, Pl. 7, Fig. 6-8.

Characters: Valves elliptic, striae in both transverse and longitudional series, straight raphe with isolated punctuate and hyaline areas appearing towards margin; $12-70 \mu \mathrm{m}$ long, 9-41 $\mu \mathrm{m}$ broad, striae $22-26$ in $10 \mu \mathrm{m}$.

Habitat: Site 1, Sundarijal, epilithic, epiphytic.

Collection no. \& date: Sundarijal, summer 1, 3; 2014-6-10; rainy 5; 2014-8-22.

Distribution in Nepal: Ponds at Kali Gandaki, 2500m., Mustang; Pisang, 3100m. and Manang Bhot Base Camp. 3500m., Mustang, (Hirano 1955; Hirano 1963).

11. Eunotia bidens (Ehr.) (Fig. 33)

References: Lange-Bertalot 1996, P. 45, Pl. 12, Fig. 5.

Characters: Valve convex dorsal side with two undulations, concave ventral side; terminal raphe fissures extend to the dorsal margin on valve face, ends broadly rostrate; striae slightly radial, parallel at center; valve 19-27 $\mu \mathrm{m}$ long, 5-9 $\mu \mathrm{m}$ broad, striae 9-13 in $10 \mu \mathrm{m}$.

Habitat: Site 1, Sundarijal, epilithic.

Collection no. \& date: Sundarijal, summer 1; 2014-6-10.

Distribution in Nepal: Maipokhari Lake, Ilam 2150m (Rai, 2005).

12. Eunotia botuliformis Wild (Fig. 34)

References: Lange-Bertalot 1993, Pl. 33, Figs. 2-7; Lange-Bertalot and Metzeltin 1996, Pl. 17, Figs. 39-42; Werum and Lange-Bertalot 2004, Pl. 5, Fig. 15; Hofmann et al. 2011, Pl. 17, Figs. 4-5, 7-8.; Lange-Bertalot et al. 2011, Pl. 128, Figs. 1-4, 11-13.;

Characters: Valve slightly curved, with sides approximately parallel; apex slightly narrow after broadly rounded; nodules visible terminals, terminal cracks very short to short raphe hardly visible, regularly spaced striae, becoming slightly more dense at the ends, but strongly radiant; $9-42 \mu \mathrm{m}$ long, 2.6 to $4 \mu \mathrm{m}$ broad, striae $14-22$ in $10 \mu \mathrm{m}$.

Habitat: Site 1, Sundarijal, epilithic.

Collection no. \& date: Sundarijal, summer 1; 2014-6-10.

Distribution in Nepal: Not reported earlier from Nepal. 
13. Eunotia minor (Kutz.) Grun. (Fig. 35)

References: Hirano, 1969, P. 16. Pl. 4, Figs. 17-18; Karthick et al. 2013, Pl. 29.

Characters: Valves slightly convex, ventral margin more or less straight; apices bluntly rounded to rostrate; widely spaced striae at the centre and more dense towards apices; 19$60 \mu \mathrm{m}$ long, $4.5-8.5 \mu \mathrm{m}$ broad, striae 9-16 in $10 \mu \mathrm{m}$.

Habitat: Site 1, Sundarijal, epilithic, epiphytic.

Collection no. \& date: Sundarijal, summer 1; 2014-6-10, rainy 5; 2014-8-22.

Distribution in Nepal: Kavrepalanchowk, a stream in Wolangchung Gola 4150m. (Hirano, 1984).

14. Gyrosigma kuetzingii (Grun.) Cl. (Fig. 36)

References: Tiffany and Britton 1952, P. 268, Pl. 66, Fig. 759.

Characters: Valves sigmoid, lanceolate, gradually attenuated to rounded poles; transverse and longitudinal striations, the former perpendicular to the middle line of the valve; $98-190$ $\mu \mathrm{m}$ long, $15-20 \mu \mathrm{m}$ broad, striae $18-22$ in $10 \mu \mathrm{m}$.

Habitat: Site 1, Sundarijal, epilithic; Site 2, Gokarna, epilithic.

Collection no. \& date: Sundarijal, summer 1; 2014-6-10; Gokarna, rainy 1; 2014-8-23.

Distribution in Nepal: A pond at Kechana, Morang 72m (Rai, 2006); a pond at Luitel Bhanjyang, Gorkha 770m (Hirano, 1955).

15. Gyrosigma scalproides (Rabh.) Cl. (Fig. 37)

References: Prasad and Srivastava 1992, P. 241, Pl. 31, Fig. 9; Agata 2009, P. 166, Pl. 24, Fig. 5

Characters: Valves sigmoid with parallel margins except at the poles where one margin strongly bent while the other slightly concave emerging into narrowly rounded ends; raphe thin, sigmoid, with distinct polar nodules; axial area narrow; central area elliptical; transverse striae thin, lineate parallel throughout the valve interrupted by more closer longitudinal striae; 45-48 $\mu \mathrm{m}$ long, $11-13 \mu \mathrm{m}$ broad, transverse striae, 24-26 in $10 \mu \mathrm{m}$, longitudinal striae, $26-28$ in $10 \mu \mathrm{m}$.

Habitat: Site 1, Sundarijal, epilithic.

Collection no. \& date: Sundarijal, summer 1; 2014-6-10.

Distribution in Nepal: Not reported earlier from Nepal.

16. Stauroneis gracilis (Ehr.) (Figs. 38-40)

Reference: Reichardt 1995, Pl. 19, Figs. 1-3; Pl. 20, Fig. 4; Metzeltin and Witkowski 1996, Pl. 10, Fig. 3; Hofmann et al. 2011, Pl. 56, Fig. 3.

Characters: Valves lanceolate; apex slightly stretched into a rostrate blunt; longitudinal area of moderate width; central area forming a butterfly shaped more or less open node with often a more or less marked asymmetry and reaching the edges of the valve; raphe oblique or curve at the center; striae radiating center, becoming the more when approaching the poles; $70-130 \mu \mathrm{m}$ long, $14-22 \mu \mathrm{m}$ broad, striae $16-22$ in $10 \mu \mathrm{m}$.

Habitat: Site 1, Sundarijal, epilithic.

Collection no. \& date: Sundarijal, summer 1; 2014-6-10.

Distribution in Nepal: A stream at Kungbachen, Panchthar 4150m, Eastern Nepal (Hirano, 1984). 
17. Stauroneis smithii Grun. (Fig. 41)

References: Krammer and Lange-Bertalot 1986, Pl. 89, Figs. 17-19; Metzeltin and Witkowski 1996, Pl. 11, Figs. 7-8; Hofmann et al. 2011, Pl. 58, Figs. 2-3.

Characters: Valves linear-lanceolate, elliptical with strong central bulge; closely to apiculate apex beaked revealing a prominent pseudo-septum with longitudinal channel parallel edges strongly silicified, quite narrow and straight, widening slightly in the vicinity of the central area; central area shaped narrow stauros with parallel edges; threadlike raphe, with well-marked central pore and distal ends hook down on the mantle; parallel striae slightly radiating; $18-39 \mu \mathrm{m}$ long, 6-11 $\mu \mathrm{m}$ broad, striae $24-27$ in $10 \mu \mathrm{m}$.

Habitat: Site 1, Sundarijal, epilithic.

Collection no. \& date: Sundarijal, summer 1; 2014-6-10.

Distribution in Nepal: Not reported earlier from Nepal.

18. Diploneis ovalis (Hilse) Cl. (Fig. 42)

Reference: Rai 2006, P. 92, Pl. 7, Fig. 14.

Characters: Valves elliptic to oval with broad, round apices; longitudinal canals narrow, the width of 1 to 3 areole, and follow the margin of the axial and central areas; central area occupied by a broad raphe, central area large and orbicular; striae uniseriate, radiate throughout; areole large, blocky and number 16-22 in $10 \mu \mathrm{m}$; 32-58 $\mu \mathrm{m}$ long, 20-33 $\mu \mathrm{m}$ broad, striae 10-14 in $10 \mu \mathrm{m}$.

Habitat: Site 1, Sundarijal, epiphytic.

Collection no. \& date: Sundarijal rainy 5; 2014-8-22.

Distribution in Nepal: Small streams flowing into Dudh Koshi river below Namche Bazar 2900-4200 m, Solukhumbu, Eastern Nepal (Suxena \& Venkateswarlu, 1968).

19. Frustulia rhomboides var. saxonica (Rab.) De Toni (Fig. 43)

Reference: Tiffany and Britton 1952, P. 245, Pl. 66, Fig. 754.

Characters: Valves rhombo-lanceolate; rectangular in girdle view, without intercalary bands; central nodule longitudinally elongated, prolonged toward each pole into two parallel ribs apically united with the polar nodule; subcapitate poles; 72-160 $\mu \mathrm{m}$ long, 14$30 \mu \mathrm{m}$ broad, transverse striations $22-30$ in $10 \mu \mathrm{m}$.

Habitat: Site 1, Sundarijal, epiphytic

Collection no. \& date: Sundarijal rainy 5; 2014-8-22.

Distribution in Nepal: Arughat Bazar, Gorkha 770m (Hirano, 1963).

20. Frustulia vulgaris (Thwaites) De Toni (Figs. 44-45)

References: Tiffany and Britton 1952, P. 245, Pl. 66, Fig. 756, Agata 2009, P. 145, Pl. 8, Figs. 10-12.

Characters: Valves elliptic to linear-lanceolate having obtuse to rostrate extrimities; striae somewhat radial; 38-80 $\mu \mathrm{m}$ long, $10-14 \mu \mathrm{m}$ broad, striae about 25 in $10 \mu \mathrm{m}$ in the middle and about 34 in $10 \mu \mathrm{m}$ at the poles.

Habitat: Site 1, Sundarijal, epilithic, epipelic; Site 2, Gokarna, epilithic, epipelic; Site 3, Pashupati, epilithic.

Collection no. \& date: Sundarijal, summer 12; 2014-6-10; Gokarna, rainy 1, 6; 2014-8-23; Pashupati, rainy 2; 2014-8-25.

Distribution in Nepal: Likhu Khola (Juttner et al., 1996) 
21. Sellaphora capitata D.G. Mann et McDonald (Fig. 46)

References: Mann, et al. 2004, P. 459-482, Figs. 4j-1, 20, 38-42; Karthick et al. 2013, Pl. 50 .

Characters: Valves narrowly elliptical with subcapitate poles; axial area narrow and straight; raphe appears as a straight line or very slightly undulate; central area is a bow-tieshaped sub-fascia and its outline is somewhat irregular due to alternate long and short striae; striae radiate, becoming parallel near the poles; $18-44 \mu \mathrm{m}$ long, 7.5-9 $\mu \mathrm{m}$ broad, striae $15-23$ in $10 \mu \mathrm{m}$.

Habitat: Site 1, Sundarijal, epilithic, epipelic, epiphytic; Site 2, Gokarna, epilithic; Site 3, Pashupati, epilithic.

Collection no. \& date: Sundarijal, summer 2, 7, 9; 2014-6-10; Sundarijal rainy 5; 2014-822; Pashupati, rainy 2; 2014-8-25.

Distribution in Nepal: Not reported earlier from Nepal.

22. Neidium ampliatum sensu Hofmann (Fig. 47)

References: Lange-Bertalot and Metzeltin 1996, Pl. 42, Figs 1-3; Hofmann et al. 2011, Pl. 54, Figs. 1-4.

Characters: Valves linear, with margins parallel, with tapered but broadly rounded apices; raphe lateral only in the middle of the valve, proximal ends deflected in opposite directions, distal raphe ends bifurcated; axial area linear, narrow, broadening to form an elliptical to quadrangular; longitudinal lines sub-marginal, broad; striae distinctly punctate, parallel except at the very ends where they are convergent; $42-100 \mu \mathrm{m}$ long, $14-23 \mu \mathrm{m}$ broad, striae $16-20$ in $10 \mu \mathrm{m}$.

Habitat: Site 1, Sundarijal, epilithic, epiphytic.

Collection no. \& date: Sundarijal, summer 12, 17; 2014-6-10.

Distribution in Nepal: Not reported earlier from Nepal.

23. Navicula escambia (R.M. Patrick) Metzeltin and Lange-Bertalot (Fig. 48)

References: Lange-Bertalot 2001, Pl. 39, Figs 1-4; Metzeltin and Lange-Bertalot 2007, Pl. 114, Figs. 18-19; Van de Vijver and Lange-Bertalot 2009, Figs. 72-80.

Characters: Valve lineo-elliptical-lanceolate; apex widely rounded; narrow longitudinal area; central area asymmetrical and variable shape: round, elliptical, rectangular or more or less lanceolate; threadlike raphe, the curved proximal terminations on the same side; shifted central nodule; striae strongly radiating across the valve; $26-54 \mu \mathrm{m}$ long, $6.5-10 \mu \mathrm{m}$ broad, striae $12-14$ in $10 \mu \mathrm{m}$.

Habitat: Site 3, Pashupati, epilithic.

Collection no. \& date: Pashupati, rainy 3; 2014-8-25.

Distribution in Nepal: Not reported earlier from Nepal.

24. Navicula radiosa Kutz. (Figs. 49-50)

References: Prasad and Srivastava 1992, P. 212, Pl. 28, Fig.4; Agata 2009, P. 175, Pl. 31, Fig. 14.

Characters: Valves linear-lanceolate, gradually attenuated towards rounded ends; raphe thin, straight, median with distinct closely set, unilaterally bent central nodules; axial area linear; central area somewhat elliptical; striae coarse lineate, curved and radiate in the middle, convergent towards the poles; 60-70 $\mu \mathrm{m}$ long, $12-14 \mu \mathrm{m}$ broad; striae 9-12 in 10 $\mu \mathrm{m}$. 
Habitat: Site 1, Sundarijal, epilithic, epipelic, epiphytic; Site 2, Gokarna, epilithic, epipelic; Site 3, Pashupati, epilithic.

Collection no. \& date: Sundarijal, summer 1-9, 12, 13, 14, 16, 18; 2014-6-10; rainy 1-18, 2014-8-22; winter 1-6, 9; 2015-2-15; Site 2, Gokarna, rainy 1-6; 2014-8-23; Pashupati, rainy 2,$3 ; 2014-8-25$.

Distribution in Nepal: Luitel Bhanjyang 770m, Gorkha; Kali Gandaki 2600m, Mustang; Tukucha moor 2640m, Mustang; Manang Bhot Base Camp 3500m, Manang (Hirano, 1963); Phewa lake 967m, Kaski (Nakanishi, 1986).

25. Navicula rhynchocephala Kutz. (Fig. 51)

References: Lange-Bertalot 2001, Pl. 9, Figs. 6-10; Siver et al. 2005, Pl. 36, Fig. 13; Hofmann et al. 2011, Pl. 29, Figs. 1-5.

Characters: Valve lanceolate, quite narrow; apex rounded, more or less sub-rostrate to capitates; longitudinal area narrow, slightly widened at the central area; central area of medium height, extended transversely to almost an elliptical shape rectangular; raphe slightly oblique; strong striae, radiant, then becoming parallel, and finally converging to poles; 40-62 $\mu \mathrm{m}$ long, $8.5-11 \mu \mathrm{m}$ broad, striae $10-12 \mu \mathrm{m}$ in $10 \mu \mathrm{m}$.

Habitat: Site 1, Sundarijal, epiphytic; Site 3, Pashupati, epilithic.

Collection no. \& date: Sundarijal, rainy 8, 2014-8-22; Pashupati, rainy 3; 2014-8-25.

Distribution in Nepal: A stream at Mewa Khola, Taplejung 4150m (Hirano, 1984).

26. Navicula rostellata Kutz. (Figs. 52-53)

References: Foged 1980, P. 654, Pl. 6, fig 7; Rai 2006, P. 89, P1. 9, Fig. 3..

Characters: Valves linear to linear-lanceolate with slightly convex margins and subrostrate apices; axial area narrow and straight; central area elliptic and slightly asymmetric; raphe straight with external proximal raphe; central nodule asymmetrically; striae bent and round towards the centre, more distant at the centre of the valve; $32-42 \mu \mathrm{m}$ long, $8-9.5 \mu \mathrm{m}$ broad, striae $12-14$ in $10 \mu \mathrm{m}$.

Habitat: Site 1, Sundarijal, epilithic; Site 2, Gokarna, epilithic, epipelic; Site 3, Pashupati, epilithic.

Collection no. \& date: Sundarijal, summer 12; 2014-6-10; Gokarna, rainy 1, 6; 2014-8-23; Pashupati, rainy 2; 2014-8-25.

Distribution in Nepal: Koshi Tappu Wildlife Reserve 75 to 100m (Udas, 2012).

27. Pinnularia cf divergens W. Smith (Figs. 54-56)

Reference: Alfinito and Lange-Bertalot 2013, P. 144, Fig. 137.

Characters: Valve linear, with straight sides and slightly tapering into broadly rounded ends; axial area broad at middle and continuously narrow towards the end; terminal fissures large, sickle shaped; raphe distinct, slightly dilated ends with distinct ends; striae short radiate at the middle, long convergent towards the ends; $45-70 \mu \mathrm{m}$ long, $8-11 \mu \mathrm{m}$ broad, striae $8-10$ in $10 \mu \mathrm{m}$. Present species are quite different having broad ends and wide middle area with short or no striae.

Habitat: Site 1, Sundarijal, epilithic; Site 2, Gokarna, epilithic, epipelic; Site 3, Pashupati, epilithic.

Collection no. \& date: Sundarijal, summer 12; 2014-6-10; Gokarna, rainy 1, 6; 2014-8-23; Pashupati, rainy 2; 2014-8-25.

Distribution in Nepal: Not reported earlier from Nepal. 
28. Pinnularia grunowii Krammer (Figs. 57-58)

Reference: Bey \& Ector 2013, P. 649, Figs. 1-2.

Characters: Valves linear, with slightly undulate outline; poles broadly capitates without distinct shoulders; raphe narrow and undulate; proximal raphe ends unilaterally bent; axial area linear, expanding broadly into the central area; striae curved and radiate; $43-53 \mu \mathrm{m}$ long, 7-8.5 $\mu \mathrm{m}$ broad, striae 8-10 in $10 \mu \mathrm{m}$.

Habitat: Site 2, Gokarna, epilithic, epipelic.

Collection no. \& date: Gokarna, rainy 6; 2014-8-23.

Distribution in Nepal: Not reported earlier from Nepal.

29. Pinnularia microstauron (Ehr.) Cl. (Fig. 59)

References: Foged 1982, P. 351, Pl. 8, Fig. 5; Agata 2009, P. 169, Pl. 26, Fig. 14.

Characters: Valves linear lanceolate with slightly constricted cuneate end; axial area broad expanded broadly into central area; central area broad; raphe thin, terminal fissures large sickle shaped; striae strongly radiate at middle, convergent towards the end; $40-40 \mu \mathrm{m}$ long, 8-9 $\mu \mathrm{m}$ broad, striae 12-14 in $10 \mu \mathrm{m}$.

Habitat: Site 1, Sundarijal, epilithic, epiphytic.

Collection no. \& date: Sundarijal, summer 12; 2014-6-10; Sundarijal, rainy 8, 2014-8-22.

Distribution in Nepal: A stream at Mewa Khola, Taplejung 4150m (Hirano, 1984).

30. Pinnularia viridis (Nitzsch) (Ehr.) (Figs. 60-62)

References: Hirano, 1972, P. 27, Pl. 8, Fig. 1-4; Karthick et al. 2013, Pl. 72.

Characters: Valves linear to elliptic-linear with slightly convex sides and broadly rounded ends; raphe complex, somewhat undulate, with a one-sided central-pore; transverse striations medianly lightly radial, polarly convergent; 50-130 $\mu \mathrm{m}$ long, 10-30 $\mu \mathrm{m}$ broad, striae 6-9 in $10 \mu \mathrm{m}$.

Habitat: Site 1, Sundarijal, epilithic, epipelic;

Collection no. \& date: Sundarijal, summer 1, 6; 2014-6-10; winter 5, 2015-2-15; Gokarna, rainy 1; 2014-8-23.

Distribution in Nepal: Koshi Basin, Sunsari 206m (Rai, 2006).

31. Gomphonema acidoclinatum Lange-Bertalot and Reichardt (Fig. 63)

Reference: Werum and Lange-Bertalot 2004, Pl. 92, Figs. 1-5, 6-11

Characters: Valves vaguely claviform closely to lanceolate, shortly heteropolar and regularly slightly curved; apex blunt and rounded triangular, more or less stretched and tapered; narrow longitudinal area and linear; unilateral central area, approximately rectangular and somewhat extended; threadlike raphe, slightly wavy, the distal ends of the same curving hook side; a well-detached stigma and in alignment with the longest streak central; generally moderately radiating striae; $20-52.5 \mu \mathrm{m}$ long, $6.5-9 \mu \mathrm{m}$ broad, striae 12 15 in $10 \mu \mathrm{m}$. .

Habitat: Site 1, Sundarijal, epilithic.

Collection no. \& date: Sundarijal, summer 1; 2014-6-10.

Distribution in Nepal: Not reported earlier from Nepal.

32. Gomphonema gandhii Karthick and Kociolek (Figs. 64-68)

Reference: Karthick, et al. 2013, Pl. 81

Characters: Valves linear-lanceolate clavate, narrowly-rounded to acuminate at the headpole; axial area broad; raphe lateral undulate; striae continuous around the headpole, 
punctate and slightly radiate to parallel; $29-43.5 \mu \mathrm{m}$ long, 5-6.5 $\mu \mathrm{m}$ broad, striae 8-12 in 10 $\mu \mathrm{m}$.

Habitat: Site 1, Sundarijal, epilithic, epipelic; Site 2, Gokarna, epilithic; Site 3, Pashupati, epilithic.

Collection no. \& date: Sundarijal, summer 1, 3, 10, 12; 2014-6-10; rainy 1, 6; 2014-8-22; winter 1, 6; 2015-2-15; Site 2, Gokarna, rainy 1; 2014-8-23; Pashupati, rainy 2; 2014-8-25.

Distribution in Nepal: Not reported earlier from Nepal.

33. Gomphonema parvulum Kutz. (Figs. 69-74)

References: Krammer and Lange-Bertalot 1991 Vol. 2/4, Pl. 76, Figs. 1-7; Kobayasi et al. 2006, Pl. 131, Figs. 1-5; Agata 2009, P. 153, Pl. 14, Figs. 8-12.

Characters: Valves very variable in shape, lanceolate to slightly clavate, or elliptical or oval, often almost naviculoide; apex rounded, sub-beaked beaked or occasionally subcapitates; narrow and straight longitudinal area; very limited central area formed unilaterally by a shorter streak or two; stigma often little detached from the median stria; raphe threadlike, often very slightly wavy; weakly radiating striations parallel; 10-36 $\mu \mathrm{m}$ long, 5-8 $\mu \mathrm{m}$ broad, striae 7-18 in $10 \mu \mathrm{m}$.

Habitat: Site 1, Sundarijal, epilithic, epiphytic; Site 2, Gokarna, epilithic; Site 3, Pashupati, epilithic.

Collection no. \& date: Sundarijal, summer 1, 3, 9, 12; 2014-6-10; rainy 1, 6; 2014-8-22; winter 1; 2015-2-15; Site 2, Gokarna, rainy 1; 2014-8-23 ; Pashupati, rainy 2; 2014-8-25.

Distribution in Nepal: A pond at Biratnagar, Morang 72m (Rai \& Rai 2005).

34. Gomphonema pseudoaugur Lange-Bert. (Figs. 75-81)

References: Agata 2009, P. 153, Pl. 14, Figs. 15-16.

Characters: Valves asymmetrical to heteropolar, symmetrical to apical axis, often broadly lanceolate in outline; apices subrostrate; raphe often slightly sinuous; single stigma present on one side of the central area; striae relatively coarse and evenly spaced; $26-55 \mu \mathrm{m}$ long, 9-13 $\mu \mathrm{m}$ broad, striae 9-11 in $10 \mu \mathrm{m}$.

Habitat: Site 1, Sundarijal, epilithic; Site 2, Gokarna, epilithic, epipelic; Site 3, Pashupati, epilithic.

Collection no. \& date: Sundarijal, summer 1, 12; 2014-6-10; winter 1; 2015-2-15; Site 2, Gokarna, rainy 1, 6; 2014-8-23 ; Pashupati, rainy 2; 2014-8-25.

Distribution in Nepal: Not reported earlier from Nepal.

35. Gomphonema rhombicum M. Schmidt (Figs. 82-83)

References: Hartley et al. 1996, Pl. 109, Fig. 13; Hofmann et al. 2011, Pl. 96, Figs. 7-10; Bey and Ector 2013, P. 940, Figs. 1-23.

Characters: Valves lanceolate, moderately heteropolar; apex weakly stretched and rounded, a little wider at the upper side than the lower side; lanceolate longitudinal area, widening a large sub-rhomboid central area; single stigma in the central nodule; raphe angular slot, sub-linear, proximal endings to the side of the curving stigma; striae short and strong, slightly sub-parallel to radiant; $36-53 \mu \mathrm{m}$ long, 5.4-7.6 $\mu \mathrm{m}$ broad, striae $9.5-12 \mu \mathrm{m}$ in $10 \mu \mathrm{m}$.

Habitat: Site 1, Sundarijal, epilithic, epipelic, epiphytic.

Collection no. \& date: Sundarijal, summer 1, 3, 6, 9, 12; 2014-6-10.

Distribution in Nepal: Not reported earlier from Nepal. 
36. Amphora ovalis Kutz. (Fig. 84)

Reference: Tiffany and Britton 1952, P. 274, Pl. 73, Fig. 855.

Characters: Valve lunate, poles blunt, ventrally concave, dorsally convex; raphe gibbous; axial area narrow; central area developed only on the ventral side; 20-140 $\mu \mathrm{m}$ long, 17-63 $\mu \mathrm{m}$ broad; transverse striations $10-13$ in $10 \mu \mathrm{m}$.

Habitat: Site 1, Sundarijal, epipelic.

Collection no. \& date: Sundarijal, summer 6; 2014-6-10.

Distribution in Nepal: Koshi Tappu Wildlife Reserve 75-100m. (Udas, 2012).

37. Cymbella aspera (Ehr.) Cl. (Fig. 86)

References: Krammer and Lange-Bertalot 1986, Pl. 131, Fig. 1.; Krammer 2002, Pl. 124, Fig. 4-6; Pl. 125, Fig. 4; Pl. 126, Fig. 1-5.; Metzeltin et al. 2009, Pl. 112, Fig. 3; Agata 2009, P. 146, Pl. 9, Figs. 1-3; Hofmann et al. 2011, Pl. 82, Figure 1.

Characteristics: Valves dorsoventral asymmetry mild to sharp, strongly convex dorsal side and ventral side with mild central bulge; apex truncated and widely rounded; median raphe almost, clearly oblique, thread like endings proximal and slightly oblique reversed near the distal ends; raphe proximal endings pore forming a rounded rather large, slightly curving side ventral and distal ends having a crack forming an angle of approximately $45^{\circ}$ dorsal side; presence of 7 to 10 stigmas at the central nodule on the ventral side differing in form of punctuation streaks; $110-200 \mu \mathrm{m}$ long, 26 to $35 \mu \mathrm{m}$ broad, striae 6.5 to 8 in $10 \mu \mathrm{m}$.

Habitat: Site 1, Sundarijal, epiphytic.

Collection no. \& date: Sundarijal, summer 9; 2014-6-10.

Distribution in Nepal: A pond at Thaple Himal, Manang 4000m (Hirano 1955); river at Triyuga V.D.C., Udayapur 152m (Rai 2006).

38. Cymbella tumida (Breb.) Van Heruck (Fig. 87)

References: Tiffany and Britton 1952, P. 278, Pl. 74, Fig. 860; Karthick et al. 2013, Pl. 91. Characters: Valves asymmetric and curved, broadly naviculoid, with rostrate poles, convex dorsal sidesand straight or slightly convex ventral sides having a median expansion; raphe excentric; axial area narrow; central area large, round, with a ventrally placed prominent isolated dot; striations transverse, radiate, punctuate; $40-105 \mu \mathrm{m}$ long, $15-23 \mu \mathrm{m}$ broad, striae 8-10 in $10 \mu \mathrm{m}$.

Habitat: Site 2, Gokarna, epilithic.

Collection no. \& date: Gokarna, rainy 1; 2014-8-23.

Distribution in Nepal: Pond at Arughat Bazar, Gorkha 710m (Hirano, 1955); Pitchhra ditch at Biratnagar, Morang 72m (Rai, 2006).

39. Cymbella turgidula Grun. (Fig. 85)

Reference: Karthick et al. 2013, Pl. 93.

Characters: Valves dorsiventral, dorsal margin strongly convex, ventral margin slightly convex; apices short and blunt, sub rostrate to rostrate truncate; axial area narrow, central area small and rounded, more developed in the dorsal side, sometimes absent in smaller specimens; raphe weakly lateral, reverse lateral at center, proximal endings rounded; 34-38 $\mu \mathrm{m}$ long, 9-11 $\mu \mathrm{m}$ broad, striae $12-16$ in $10 \mu \mathrm{m}$.

Habitat: Site 1, Sundarijal, epilithic; Site 2, Gokarna, epipelic; Site 3, Pashupati, epilithic.

Collection no. \& date: Sundarijal, summer 1, 12; 2014-6-10; rainy 1; 2014-8-22; Site 2, Gokarna, rainy 6; 2014-8-23; Pashupati, rainy 2; 2014-8-25. 
Distribution in Nepal: Not reported earlier from Nepal.

40. Encyonema hustedtii Krammer (Fig. 88)

Reference: Karthick et al. 2013, Pl. 99

Characters: Valves dorsiventral, semi-elliptic, dorsal strongly convex, ventral bulbous at middle; apex rounded, relatively acute; axial area moderately to strongly displace anteriorly; central area absent, but with narrow widening at dorsal region; raphe moderately wide, straight to slightly curve; striae punctuate, broadly spaced in middle and coarser at ends; 38-62.0 $\mu \mathrm{m}$ long, 9.5-13 $\mu \mathrm{m}$ broad, striae 7-12 in $10 \mu \mathrm{m}$.

Habitat: Site 1, Sundarijal, epilithic; Site 2, Gokarna, epilithic.

Collection no. \& date: Sundarijal, summer 1; 2014-6-10; Gokarna, rainy 1; 2014-8-23.

Distribution in Nepal: Not reported earlier from Nepal.

41. Encyonema ventricosum (Kutz.) Grun. (Fig. 89)

References: Krammer 1997, Pl. 6, Figs. 11-12; Pl.7, Figs. 3-5; Agata 2009, P. 150, Pl. 12, Figs. 8, 9, 12; Hofmann et al. 2011, Pl. 87, Figs. 18-22.

Characters: Valve strongly convex dorsal side and ventral more or less in its bulging portion median or almost rectilinear; apex sub-capitate, slightly curved ventral side; raphe threadlike linear, distal ends curved ventral side; striae parallel in the center, becoming more or less radiant dorsal side, and convergent only the ventral side ends; $8.5-28 \mu \mathrm{m}$ long, 4-7 $\mu \mathrm{m}$ broad, striae 12-18 in $10 \mu \mathrm{m}$.

Habitat: Site 1, Sundarijal, epilithic.

Collection no. \& date: Sundarijal, summer 12; 2014-6-10.

Distribution in Nepal: Not reported earlier from Nepal.

42. Epithemia adnata (Kutz.) Breb. (Figs. 90-91)

Reference: Bey and Ector 2013, P. 1124, Figs. 1-14; Karthick et al. 2013, Pl. 102-103.

Characters: Valves dorsiventral, dorsal margin convex, ventral margin straight or weakly concave; apex broadly rounded; raphe branches bi-arcuate towards dorsal side and end approximately in the centre of the valve; raphe supported by fibulae; 31-65 $\mu \mathrm{m}$ long, 7-11 $\mu \mathrm{m}$ broad, striae $12-13$ in $10 \mu \mathrm{m}$.

Habitat: Site 1, Sundarijal, epilithic.

Collection no. \& date: Sundarijal, summer 12; 2014-6-10

Distribution in Nepal: Not reported earlier from Nepal.

43. Epithemia sorex Kutz. (Fig. 92)

References: Foged 1980, P. 441, Pl. 6, Fig. 7; Hadi et al. 1984, P. 536, Pl. 5, Fig. 83; Pl 12, Figs. 213-214; Karthick et al. 2013, Pl. 104.

Characters: Valves strongly dorsiventrally arched, taper towards rounded to rostrate or capitates poles; raphe canal lies against the ventral margin at distal ends of the valve and arches toward the dorsal margin in the valve center; central arch of the canal reaches almost to the dorsal margin; striae punctuate, there are 2 (sometimes 3 ) striae between costae; 25 $52 \mu \mathrm{m}$ long, 10-13.5 $\mu \mathrm{m}$ broad, costae 5 in $10 \mu \mathrm{m}$, striae $10-12$ in $10 \mu \mathrm{m}$.

Habitat: Site 3, Pashupati, epilithic.

Collection no. \& date: Pashupati, rainy 2; 2014-8-25.

Distribution in Nepal: Koshi Tappu Wildlife Reserve 75-100m (Udas, 2012). 
44. Nitzschia linearis (Agardh) Smith (Figs. 93-94)

References: Hirano 1971, P. 99, Pl. 7, Fig.24; Agata 2009, P. 183, Pl. 37, Figs. 1-5; Karthick et al. 2013, Pl. 117.

Characters: Valve linear to lanceolate with capitates to rostrate poles, keel bearing side slightly concave; raphe marginal; central area distinct; 100-125 $\mu \mathrm{m}$ long, 2.5-3.5 $\mu \mathrm{m}$ broad; striae fine, $28-30$ in $10 \mu \mathrm{m}$.

Habitat: Site 1, Sundarijal, epilithic, epipelic, epiphytic; Site 2, Gokarna, epilithic, epipelic; Site 3, Pashupati, epilithic.

Collection no. \& date: Sundarijal, summer 2, 6, 7, 8, 12; 2014-6-10; winter 1, 6; 2015-215; Gokarna, rainy 2, 6; 2014-8-23; Pashupati, rainy 1; 2014-8-25.

Distribution in Nepal: A stream at Kungbachen, Panchthar 4150m (Hirano, 1984).

45. Nitzschia palea (Kutz.) W. Smith (Figs. 95-99)

References: Prowse 1962, P. 68, Pl. 19, Figs. K, P; Hirano 1974, Pl. 5, Fig 17; Prasad and Srivastava 1992, P. 288, Pl. 35, Fig 5.

Characters: Valves linear-lanceolate, gradually narrowing towards slightly produced subrostrate ends; keel punctuate distinct, slightly elongated, irregularly placed; striae indistinct; $52 \mu \mathrm{m}$ long, $3.5 \mu \mathrm{m}$ broad; keel punctae, $13-14$ in $10 \mu \mathrm{m}$; striae more than 35 in $10 \mu \mathrm{m}$.

Habitat: Site 1, Sundarijal, epilithic, epiphytic; Site 2, Gokarna, epilithic, epipelic; Site 3, Pashupati, epilithic.

Collection no. \& date: Sundarijal, summer 2, 7, 8, 12; 2014-6-10; Gokarna, rainy 2, 6; 2014-8-23; Pashupati, rainy 1; 2014-8-25.

Distribution in Nepal: A stream at Mewa river, Taplejung 4150m (Hirano, 1984); Koshi Tappu Wildlife Reserve 75-100m (Udas, 2012).

46. Surirella angusta Kutz. (Figs. 100-101)

References: Krammer and Lange-Bertalot 1988, vol. 2/2, Pl. 133, Figs. 6, 7, 8-11, 12,13; Lange-Bertalot and Metzeltin 1996, Pl. 106, Fig. 15; Metzeltin et al. 2005, Pl. 221, Figs 17; Levkov et al. 2007, Pl. 203, Fig. 1; Hofmann et al. 2011, Pl. 131, Figs. 1-5.

Characters: Valve isopolar, weakly heteropolar, linear in shape and sides parallel but becoming lineo-lanceolate to lanceolate in smaller individuals; apex beaked sometimes subbeaked; pseudo-raphe narrow and straight; striae sometimes irregular, low contrast; 15-70 $\mu \mathrm{m}$ long, $6.5 \mu \mathrm{m}$ broad, striae $20-28$ in $10 \mu \mathrm{m}$.

Habitat: Site 2, Gokarna, epilithic, epipelic.

Collection no. \& date: Gokarna, rainy 2, 6; 2014-8-23.

Distribution in Nepal: Not reported earlier from Nepal.

47. Surirella linearis W. Smith (Figs. 102-105)

References: Hirano, 1972, Pl. 13, Fig. 1; Tiffany and Britton 1952, P. 294, Pl. 79, Fig. 920. Characteristics: Valves linear with parallel or slightly convex sides, isopolar, and bluntly rounded nearly cuneate poles, $20-125 \mu \mathrm{m}$ long, 9-25 $\mu \mathrm{m}$ broad, costae 2-5 in $10 \mu \mathrm{m}$.

Habitat: Site 1, Sundarijal, epilithic, epipelic, epiphytic.

Collection no. \& date: Sundarijal, summer $2,4,5,7,8,12$; 2014-6-10; rainy 1, 5; 2014-822; winter 1, 6, 9; 2015-2-15

Distribution in Nepal: Ponds at Luitel Bhanjyang, Gorkha and Phewa tal, Kaski (Hirano, 1955); Streams of Gajurmukhi, Ilam (Rai et al., 2008). 

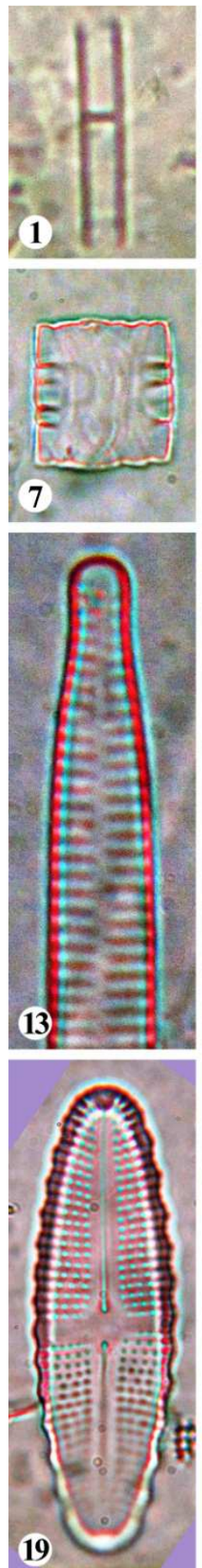

19
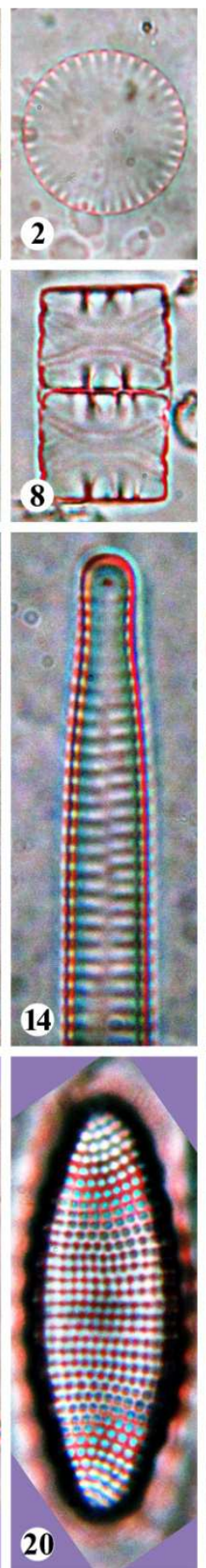
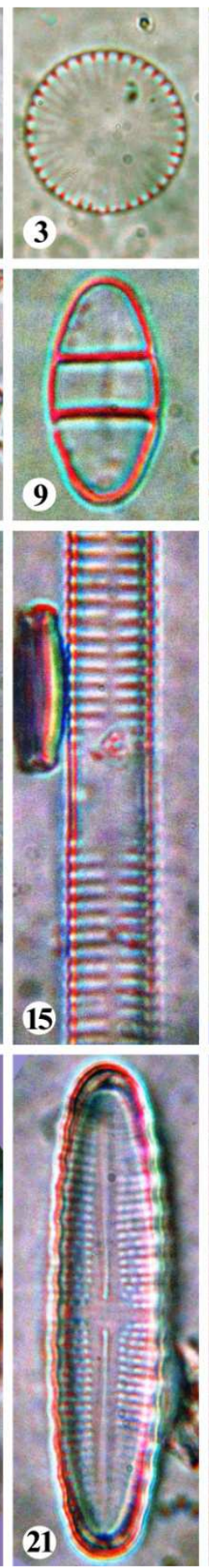
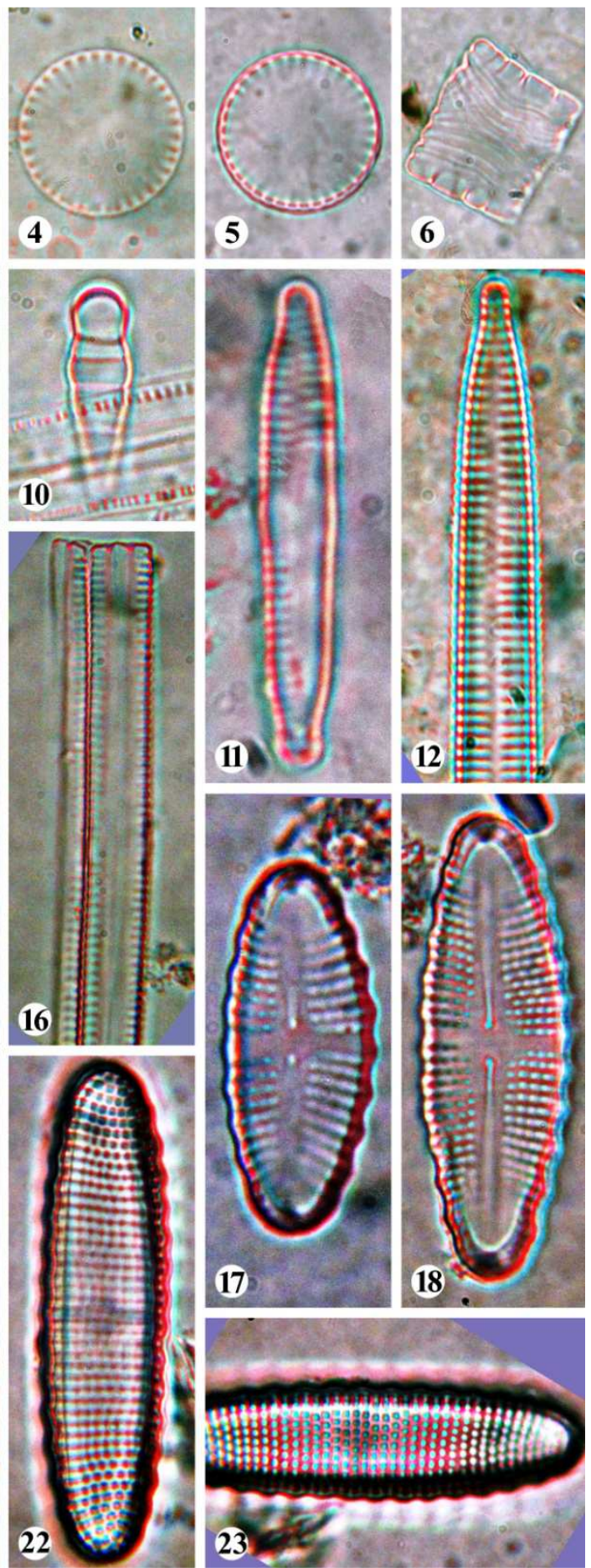
mesodon (girdle view), 9. D. hiemale var. mesodon (valve view), 10. Meridion circulare var. constrictum, 11. Fragilaria vaucheriae, 12-15. Ulnaria ulna (valve view), 16. U. ulna (girdle view), 17-23. Achnanthes crenulata (valve view) 

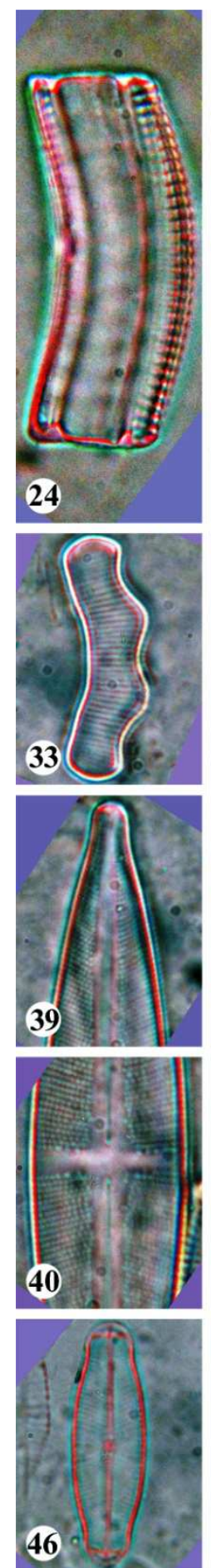
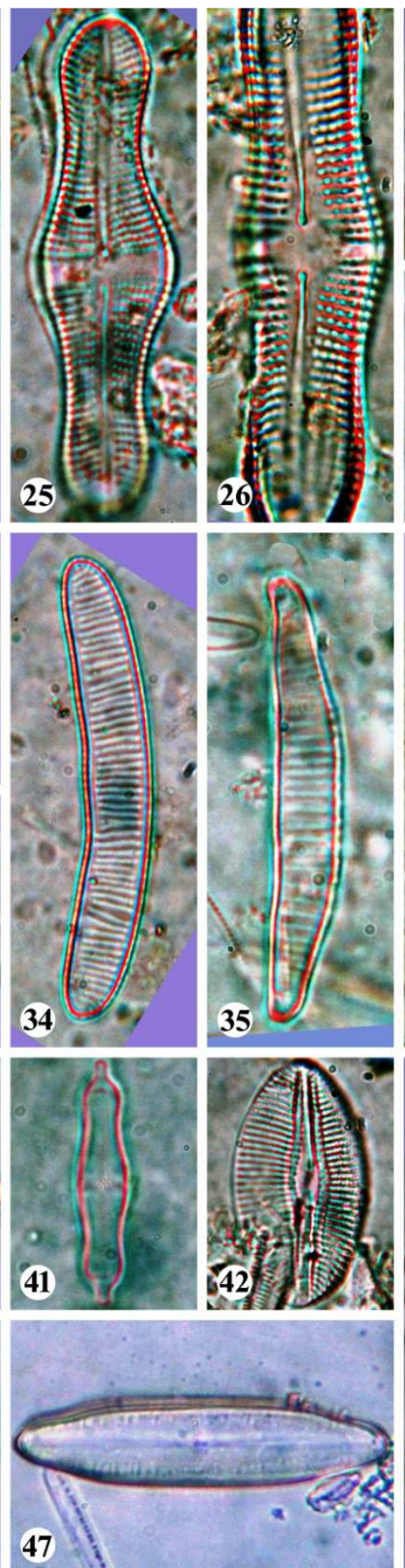
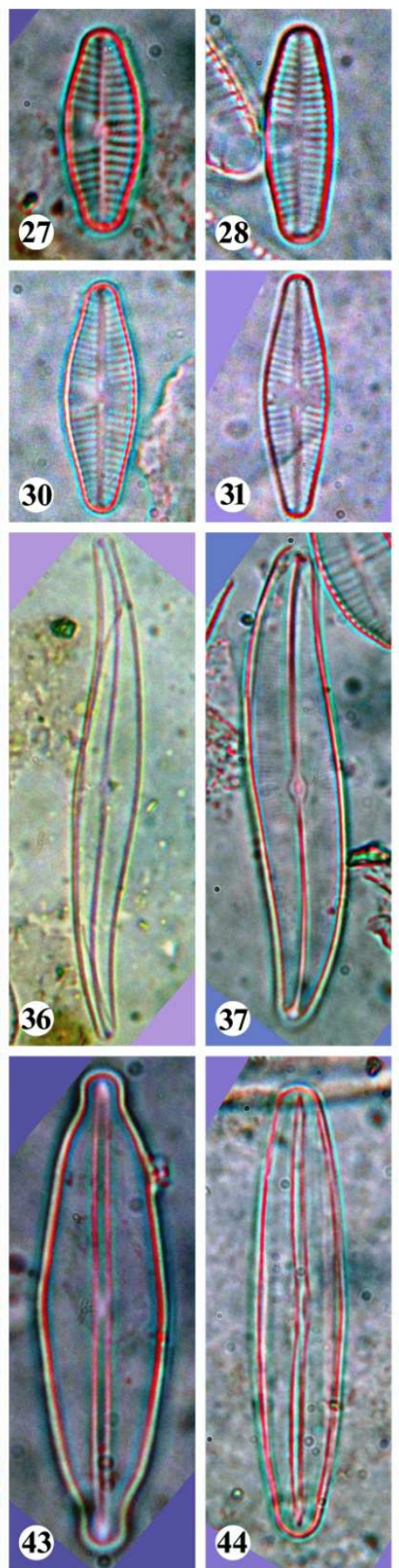
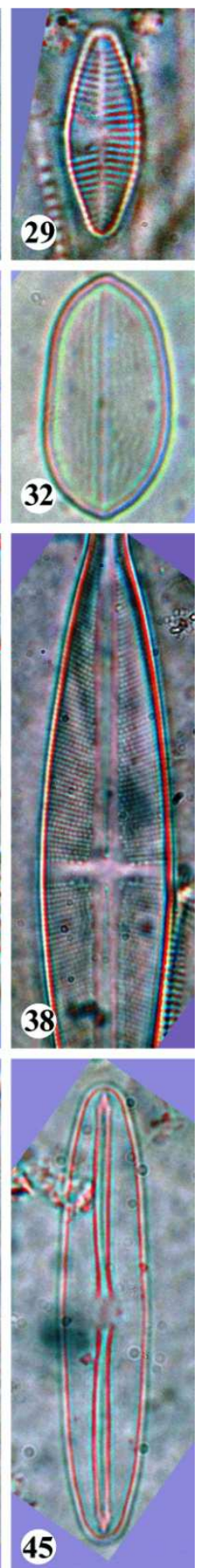

Figures: 24. Achnanthes crenulata (girdle view), 25-26. A. inflata, 27-31. Planothidium lanceolatum, 32. Cocconies placentula, 33. Eunotia bidens, 34. E. botuliformis, 35. E. minor, 36. Gyrosigma kuetzingii, 37. G. scalproides, 38-40. Stauroneis gracilis, 41. S. smithii, 42. Diploneis ovalis, 43. Frustulia rhomboids var. saxonica, 44-45. F. vulgaris, 46. Sellaphora capitata, 47. Neidium ampliatum 

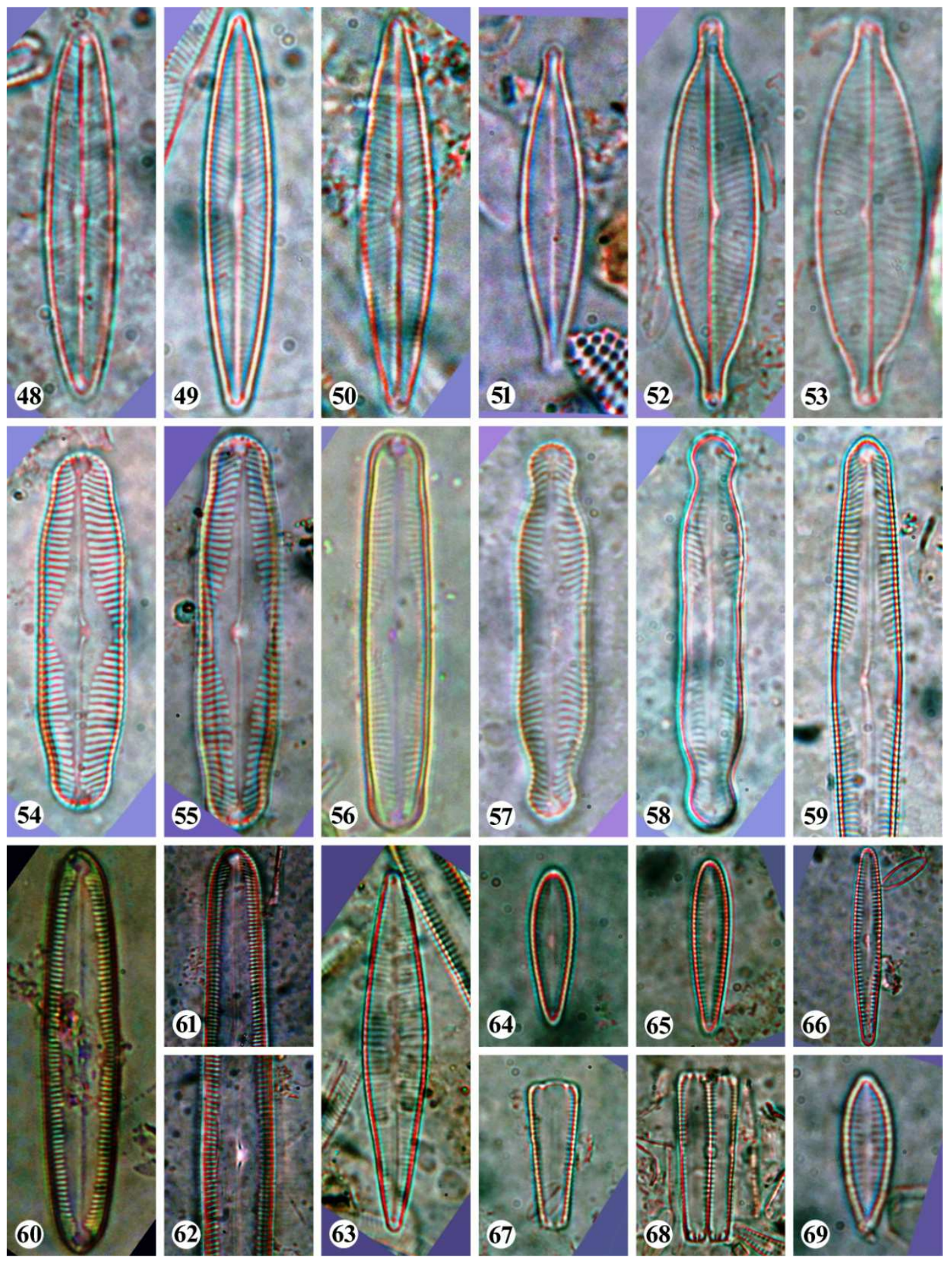

Figures: 48. Navicula escambia, 49-50. N. radiosa, 51. N. rhyncocephala, 52-53. N. rostellata, 5456. Pinnularia ef divergens, 57-58. P. grunowii, 59. P. microstauron, 60-62. P. viridis, 63. Gomphonema acidoclinatum, 64-68. G. gandhii, 69. G. parvulum 

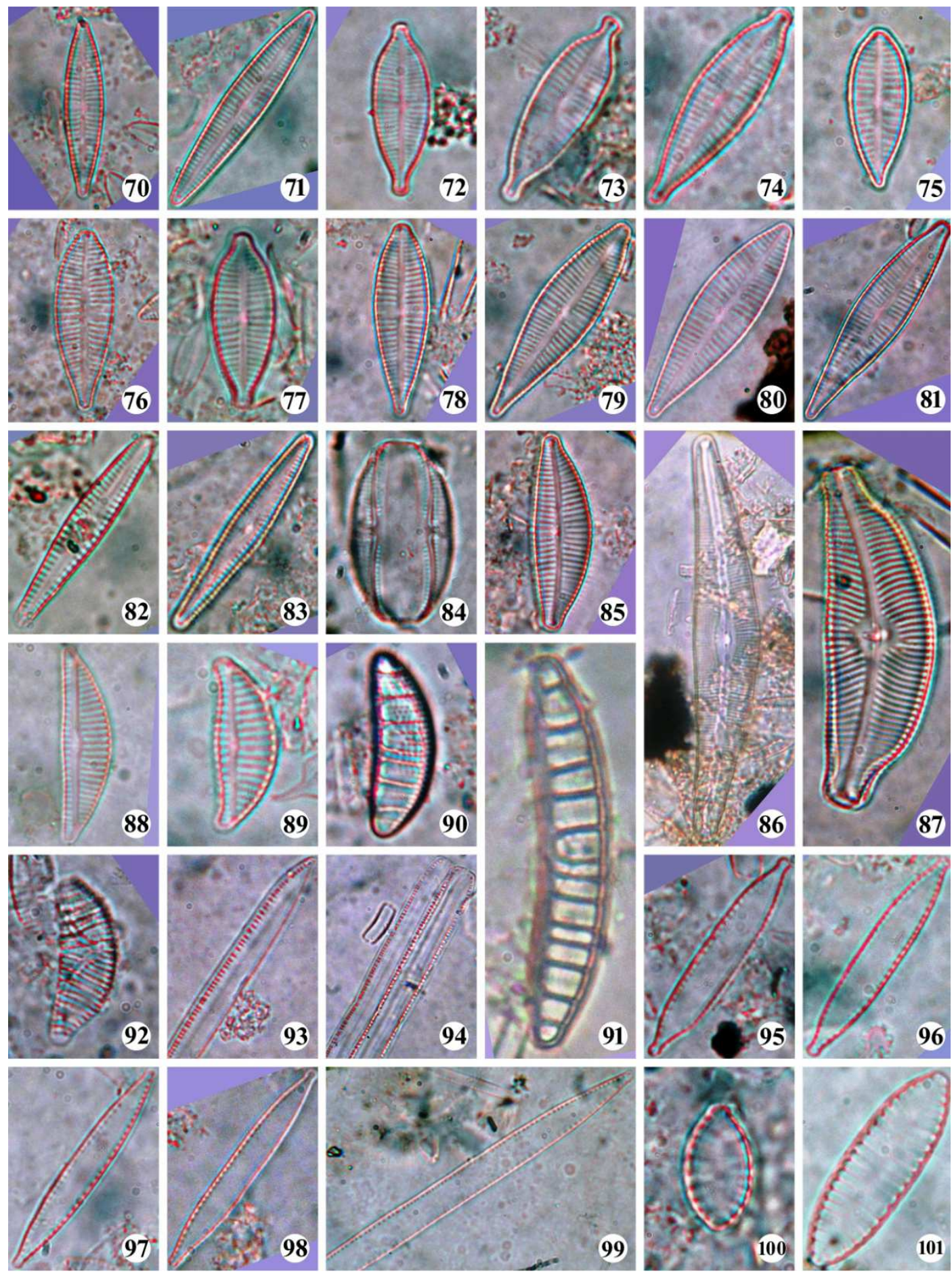

Figures: 70-74. Gomphonema parvulum, 75-81. G. pseudoaugur, 82-83. G. rhombicum, 84. Amphora ovalis 85. Cymbella turgidula, 86. C. aspera, 87. C. tumida, 88. Encyonema hustedtii, 89. E. ventricosum, 90-91. Epithemia adnata, 92. E. sorex, 93-94. Nitzschia linearis, 95-99. N. palea, 100-101. Surirella angusta 

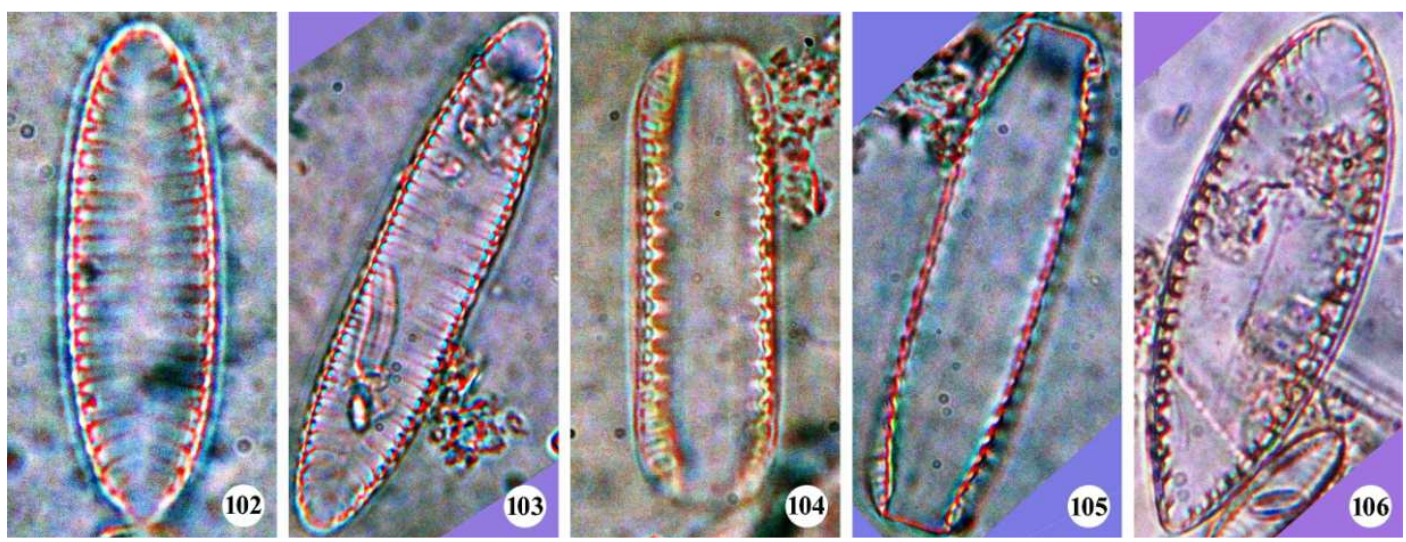

Figures: 102-105. Surirella linearis, 106. S. splendida

48. Surirella splendida (Ehr.) Kutz. (Fig. 106)

References: Krammer and Lange-Bertalot 1988, vol. 2/2, Pl. 158, Figs. 1, 1A; LangeBertalot and Metzeltin 1996, Pl. 72, Fig. 3.

Characters: Valve oval, sharply heteropolar, the larger pole rounded, the narrowest pole truncated, triangle rounded; relatively broad pseudo-raphe to lanceolate; sub-parallel ribs parallel to the center, becoming radiant at poles; $75-250 \mu \mathrm{m}$ long, $40-70 \mu \mathrm{m}$ broad, wings $12-25$ in $100 \mu \mathrm{m}$

Habitat Site 1, Sundarijal, epilithic, epipelic.

Collection no. \& date: Sundarijal, summer 2, 4.

Distribution in Nepal: Phewa lake, Kaski 967m; a stream at Ankhu Khola 630m and moist soil at Arughat Bazar 640m, Gorkha (Hirano, 1955).

A total 48 diatom taxa were reported from Bagmati river belonging to 12 families and 25 genera. Gomphonema was the richest genera represented by 5 taxa. In the present study, 20 diatoms were reported as new to Nepal from Bagmati River viz., Fragilaria vaucheriae, Achnanthes inflata, Planothidium lanceolatum, Eunotia botuliformis, Gyrosigma scalproides, Stauroneis smithii, Sellaphora capitata, Neidium ampliatum, Navicula escambia, Pinnularia cf divergens, P. grunowii, Gomphonema acidoclinatum, G. gandhii, G. pseudoaugur, G. rhombicum, Cymbella turgidula, Encyonema hustedtii, E. ventricosum, Epithemia adnata, and Surirella angusta. Further extensive studies are essential to understand more new and interesting diatoms of this river.

\section{Acknowledgement}

Authors are thankful to the Head, Department of Botany, Post Graduate Campus, Tribhuvan University, Biratnagar for laboratory facilities.

\section{References}

Agata, Z.W. 2009. The diatoms of Koblyanka stream near Krakow. Polish Botanical Journal 54(2): 129-330.

Alfinito, S. \& H. Lange-Bertalot 2013. Contribution to the knowledge of the freshwater algae of Sierra Leone (Tropical West Africa): Diatoms from Loma Mountains and Bumbuna Falls, the Northern Province. Biodiversity Journal 4(1): 135-178. 
Aryal, S. \& P. Lacoul 1996. Water quality and diversity of diatoms in Punyamati river, Nepal. Ecoprint 3(1): 45-49.

Bey, M-Y. \& L. Ector 2013. Atlas of river diatoms the Rhone Alpes region. Gabries Lippmann.

Bradbury, J. 2004. Nature's nanotechnologists: Unveiling the secrets of diatoms. PLoS Biology 2(10): 1512-1515.

Drum, R.W. \& R. Gordon 2003. Star Trek replicators and diatom nanotechnology. Trends Biotechnology 21: 325-328.

Foged, N. 1980. Diatoms in Egypt. Nova Hedwigia 33(1-4): 629-675-707.

Foged, N. 1982. Diatoms in Asklepieion, Pergamen, Turkey. Nova Hedwigia 36(2-4): 587-620.

Hadi, R.A.M., A.A. Al-saboonchi \& A.K.Y. Haroon 1984. Diatoms of the Shatt al-Arab river, Iraq. Nova Hedwiga 39(3-4): 513-544-557.

Hartley, B., H.G. Barber, J.R. Carter \& P.A. Sims 1996. An atlas of British diatoms. Biopress Ltd, England.

Hayashi, T. 2011. Monospecific planktonic diatom assemblages in the Paleo-Kathmandu Lake during the middle Brunhes Chron: implications for the paradox of the plankton. Palaeogeography, Palaeoclimatology, Palaeoecology 300(1-4): 46-58.

Hayashi, T. \& Y. Tanimura 2015. Morphological variability of Cyclostephanos ramosus sp. nov. from Pleistocene sediments of the Paleo-Kathmandu Lake, Nepal. Diatom 31: 1-11. DOI: $10.11464 /$ diatom.31.1

Hayashi, T. \& Y. Tanimura 2015. Two new Cyclostephanos species, C. nepalensis and C. pseudonepalensis (Bacillariophyta), from middle Pleistocene sediments of the PaleoKathmandu Lake, Nepal. Nova Hedwigia 101(1-2): 191-204.

Hayashi, T., Y. Tanimura \& H. Sakai 2007. Puncticulata versiformis sp. nov. and Cyclotella kathmanduensis sp. nov. (Bacillariophyta), new fossil species from middle pleistocene lacustrine sediments, Kathmandu, Nepal Himalaya. Journal of Phycology 43: 304-318.

Hayashi, T., Y. Tanimura, Y. Kuwahara, T. Maki, R. Fujii, M. Mampuku, T. Yamanaka, M. Ohno \& H. Sakai 2012. Paleosynecology of diatoms in the Paleo-Kathmandu lake during the middle to late Pleistocene: Variation in productivity and species diversity of planktonic and benthic assemblages. Journal of Geography (Chigaku Zasshi) 121(6): 962-985. DOI: 10.5026/ jgeography.121.962

Hickel, B. 1973. Phytoplanktons in two ponds in Kathmandu valley, Nepal. Int. Rev. ges Hydrobiol. 58(6): 835-842

Hirano, M. 1955. Fresh water algae. In: Fauna and Flora of Nepal Himalaya (Ed. H. Kihara). Fauna and Flora Research Society, Kyoto University, Kyoto, Japan. pp. 5-42.

Hirano, M. 1963. Fresh water algae from the Nepal Himalaya, collected by a member of the Japanese Climbing Expedition. Contr. Biol. Lab., Kyoto University, Japan. 16: 1-23.

Hirano, M. 1969. Freshwater Algae from Rangtang Himal, Nepal Himalaya. Contribution from the Biological Laboratory, Kyoto University, Japan. 22: 1-42.

Hirano, M. 1971. Freshwater algae of Northwestern Himalaya. Contribution from the Biological Laboratory, Kyoto University, Japan. 23(2): 81-100.

Hirano, M. 1972. Diatoms from the Hida Mountain Range in the Japan Alps. Contribution from the Biological Laboratory, Kyoto University, Japan. 24(1): 10-30.

Hirano, M. 1974. Freshwater algae from North Borneo. Contributions from the Biological Laboratory, Kyoto University 24(3): 122-144. 
Hirano, M. 1984. Fresh water algae from East Nepal. Study report of Baika Junior College 32: 197-215.

Hofmann, G., M. Werum \& H. Lange-Bertalot 2011. Diatomeen im Süßwasser-Benthos von Mitteleuropa. Koeltz Scientific Books, Königstein. 908p, 133 pl., 3522 figs.

IPCC 2007. Climate change 2007. Synthesis Report. Core Writing Team. 104p.

Johnson, R.C. 2009. Diatoms could triple solar cell efficiency. EE Times.

Juettner, I., H. Rothfritz \& S.J. Ormerod 1996. Diatoms as indicators of river quality in the Nepalese middle hills with consideration of the effects of habitat-specific sampling. Freshwater Biology 36: 475-486.

Karthick, B. P.B. Hamilton \& J.P. Kociolek 2013. An illustrated guide to common diatoms of Peninsular India. Gubbi Labs, Gubbi. 206p.

Kobayasi, H., M. Idei, S. Mayama, T. Nagumo \& K. Osada 2006. H. Kobayasi's Atlas of Japanese diatoms based on electron microscopy, vol. 1. Uchida Rokakuko Publishing Co., Tokyo. $59+533$ p., 180 pls.

Krammer, K. \& H. Lange-Bertalot 1986. Bacillariophyceae 1. Teil: Naviculaceae. In: Suesswasser-flora von Mitteleuropa (Eds. H. Ettl et al.). VEB Gustav Fisher Verlag, Jena 2(1): 1-876, 206 pls., 2976 figs.

Krammer, K. \& H. Lange-Bertalot 1988. Bacillariophyceae. 2. Teil: Bacillariaceae, Epithemiaceae, Surirellaceae. In: Süsswasserflora von Mitteleuropa, band 2/2 (Eds. H. Ettl, J. Gerloff, H. Heynig \& D. Mollenhauer). VEB Gustav Fischer Verlag, Jena 596p.

Krammer, K. \& H. Lange-Bertalot 1991. Bacillariophyceae 4. Teil: Achnanthaceae, Kritische Erganzungen zu Navicula (Lineolatae) und Gomphonema Gesamt literature verzeichnis Teil 1-4. In: Suesswasserflora von Mitteleuropa (Eds. H. Ettl et al.). VEB Gustav Fisher Verlag, Jena 2(4): 1-437, 88 pls., 2048 figs.

Krammer, K. 1997. Die cymbelloiden Diatomeen - Eine Monographie der weltweit bekannten Taxa. Teil 1. Allgemeines und Encyonema Part. Bibliotheca Diatomologica 36: 382p.

Krammer, K. 2002. Cymbella. In: Diatoms of Europe, diatoms of the European inland waters and comparable habitats (Ed. H. Lange-Bertalot). A.R.G. Gantner Verlag K.G. 3: 1-584.

Lange-Bertalot, H. \& D. Metzeltin 1996. Iconographia Diatomologica. Annotated Diatom Micrographs, vol. 2. Ecology, Diversity, Taxonomy. Koeltz Scientific Books. Königstein, Germany, $390 \mathrm{p}$.

Lange-Bertalot, H. 1980. Zur taxonomischen Revision einiger ökologisch wichtiger "Navicula lineolatae" Cleve. Die Formenkreise um Naviculae lanceolata, N. viridula, N. cari. Cryptogamie Algologie 1(1): 29-50.

Lange-Bertalot, H. 1993. 85 neue taxa und über 100 weitere neu definierte Taxa ergänzend zur Süsswasserflora von Mitteleuropa, vol. 2/1-4. Bibliotheca Diatomologica 27: 164 p., 134 pls.

Lange-Bertalot, H. 1996. Iconographica Diatomologica. Anotated diatom micrographs, vol. 2 , Koeltz Scientific Book.

Lange-Bertalot, H. 2001. Navicula sensu stricto, 10 genera seperated from Navicula sensu lato, Frustulia. In: Diatoms of Europe, diatoms of the European inland waters and comparable habitats (Ed. H. Lange-Bertalot). A.R.G. Gantner Verlag K.G. 2: 1-526.

Lange-Bertalot, H., M. Bak, A. Witkowski \& N. Tagliaventi 2011. Eunotia and some related genera. In: Diatoms of Europe. Diatoms of the European inland water and comparable 
habitats (Ed. H. Lange-Bertalot). A.R.G. Gantner Verlag K.G. 6: 747, 237 plates, 5053 figs.

Levkov, Z., S. Krstic, D. Metzeltin \& T. Nakov 2007. Diatoms of lakes, Prespa and Ohrid, about 500 taxa from ancient lake system. In: Iconographia Diatomologica. Annotated Diatom Micrographs. Biogeography, Ecology, Taxonomy (Ed. H. Lange-Bertalot). A.R.G. Gantner Verlag K.G. 16: 613.

Mann, D.G. \& S.J.M. Droop 1996. Biodiversity, biogeography and conservation of diatoms. Hydrobiologia 336: 19-32.

Mann, D.G., S.M. McDonald, M.M. Bayer, S.J.M. Droop, V.A. Chepurnov, R.E. Loke, A. Ciobanu, \& J.M.H. du Buf 2004. The Sellaphora pupula species complex (Bacillariophyceae): morphometric analysis, ultrastructure and mating data provide evidence for five new species. Phycologia 43(4): 459-482, 52 figs., 4 tables.

Metzeltin, D. \& A. Witkowski 1996. Diatomeen der Bären-Insel. Süsswasser- und marine Arten. In: Iconographia Diatomologica. Annotated Diatom Micrographs, vol. 4. Taxonomy (Ed. H. Lange-Bertalot). Koeltz Scientific Books, Königstein, Germany 4: 3217

Metzeltin, D. \& H. Lange-Bertalot 2007. Tropical diatoms of South America II. Special remarks on biogeography disjunction. In: Iconographia Diatomologica. Annotated Diatom Micrographs, vol. 18. Diversity-Taxonomy-Biogeography (Ed. H. Lange-Bertalot). A.R.G. Gantner Verlag K.G. 18: 1-877.

Metzeltin, D., H. Lange-Bertalot \& F. Garcia-Rodriguez 2005. Diatoms of Uruguay. Compared with other taxa from South America and elsewhere. In: Iconographia Diatomologica. Annotated Diatom Micrographs. Vol. 15. Taxonomy-Biogeography-Diversity (Ed. H. Lange-Bertalot). A.R.G. Gantner Verlag K.G. 15: 736.

Metzeltin, D., H. Lange-Bertalot \& S. Nergui 2009. Diatoms in Mongolia. In: Iconographia Diatomologica. Annotated Diatom Micrographs, vol. 20 (Ed. H. Lange-Bertalot). A.R.G. Gantner Verlag K.G. 20: 3-686.

Nakanishi, M. 1986. Limnological study in Phewa, Begnas and Rupa lakes. In: Studies on distribution, adaptation and evolution of microorganisms in Nepal Himalayas (Second report) (Ed. Y. Ishida). Ministry of Education, Science and Culture, Kyoto, Japan. pp. 313.

PCER, ANSP 1988. Diatom cleaning by nitric acid digestion. Protocol no. P-13-02. Patrick Center for Environmental Research, Academy of Natural Sciences of Philadelphia. http://diatom. acnatsci.org/nawqa/pdfs/

Prasad, B.N. \& M.N. Srivastava 1992. Fresh water algal flora of Andaman and Nicobar Islands, vol. I. B. Singh \& M.P. Singh Publ., Dehra Dun, India. 369p.

Prescott, G.W. 1969. The algae: A review, Thomas Nelson \& Sons, London.

Prowse, G.A. 1962. Diatoms of Malayan fresh waters. The garden's bull., Singapore 19(1): 1105.

Prygiel, J., B.A. Whitton \& J. Bukowska 1999. Use of the algae for monitoring rivers III. Agence de l'Eau ArtoisPicardie, Douai Cedex.

Rai, S.K. 2005. Preliminary report of diatoms from Maipokhari lake, Ilam, Nepal. Our Nature 3(1): 26-30.

Rai, S.K. 2006. Taxonomic studies on some freshwater diatoms from the Eastern Tarai Region, Nepal. Our Nature 4: 10-19. 
Rai, S.K. and R.K. Rai 2005. Some bacillariophycean algae from Biratnagar, Nepal. Ecoprint 12: $71-76$.

Rai, S.K., B.R. Subba \& K.P. Limbu 2008. Fresh water algae from running streams of Gajurmukhi VDC, Ilam, Nepal. Our Nature 6: 80-81

Ramachandra, T.V., D.M. Mahapatra, B. Karthick \& R. Gordon 2009. Milking diatoms for sustainable energy: biochemical engineering versus gasoline-secreting diatom solar panels. Industrial and engineering chemistry research, Complex Materials II special issue, October 48(19): 8769-8788.

Reichardt, E. 1995. Die Diatomeen (Bacillariophyceae) in Ehrenberg's material von Cayenne, Guyana Gallica 1843. In: Iconographia Diatomologica. Annotated Diatom Micrographs, vol. 1. Taxonomy (Ed. H. Lange-Bertalot). Koeltz Scientific Books. Königstein, Germany 1: 7-99.

Rumrich, U., H. Lange-Bertalot \& M. Rumrich 2000. Diatoms of the Andes. From Venezuela to Patagonia/Tierra del Fuego and two additional contributions. In: Iconographia Diatomologica. Annotated Diatom Micrographs, vol. 9. Phytogeography-DiversityTaxonomy (Ed. H. Lange-Bertalot). Koeltz Scientific Books, Königstein, Germany 9: 673.

Shrestha, B. \& J.D. Manandhar 1983. Contribution to the algal flora of Kathmandu valley. $J$. Inst. Sci. Tech. (Nepal) 6: 1-6.

Simkhada, B. \& I. Jüttner 2006. Diatoms in ponds and small lakes of the Kathmandu valley, Nepal- relationships with chemical and habitat characteristics. Arch. Hydrobiol. 166(1): 41-65. DOI: 10.1127/0003-9136/2006/0166-0041

Siver, P.A., P.B. Hamilton, K. Stachura-Suchoples \& J.P. Kociolek 2005. Diatoms of North America: The freshwater flora of Cape Cod, Massachusetts, U.S.A. In: Iconographia Diatomologica. Annotated Diatom Micrographs. Diatoms of North America (Ed. H. Lange-Bertalot). A.R.G. Gantner Verlag K.G. 14: 463, 94 pls.

Stoermer, E.F. \& J.P. Smol 1999. The diatoms: applications for the environmental and Earth Sciences. Cambridge University Press, Cambridge.

Suxena, M.R. \& V. Venkateswarlu 1968. Algae of the Cho Oyu (E. Himalaya) Expedition-I. Bacillariophyceae. Hydrobiologia 32: 1-26.

Tiffany, L.H. \& M.E. Britton 1952.The algae of Illinois. Hafner Publishing Company, New York. 407p.

Treguer, P., D.M. Nelson, A.J. Van Bennekom, D.J. DeMaster, A. Lyenaert \& B. Queguiner 1995. The silica balance in the World Ocean: A re-estimate. Science 268(5209): 375-379.

Udas, S. 2012. Studies on algal diversity of Koshi Tappu Wetland, Sunsari. M.Sc. Thesis, Department of Botany, P.G. Campus, T.U., Biratnagar.

Van de Vijver, B. \& H. Lange-Bertalot 2009. New and interesting Navicula taxa from western and northern Europe. Diatom Research 24(2): 431-441.

Werum, M. \& H. Lange-Bertalot 2004. Diatoms in springs from Central Europe and elsewhere under the influence of hydrologeology and anthropogenic impacts. In: Iconographia Diatomologica. Annotated Diatom Micrographs, vol. 13. Ecology-Hydrology-Taxonomy (Ed. H. Lange-Bertalot). A.R.G. Gantner Verlag K.G. 13: 3-417, 105 pls. 